\title{
Feeding difficulties during the neonatal period
}

\author{
Luigi Corvaglia, Silvia Martini \\ From 71st Congress of the Italian Society of Pediatrics. Joint National Meeting SIP, SIMGePeD, Study Group \\ on Pediatric Ultrasoun, SUP Study Group on Hypertension \\ Rome, Italy. 4-6 June 2015
}

Feeding difficulties (FD) are a major issue in neonatology, as they could hamper the assessment of an adequate enteral nutrition, delay hospital discharge and lead to breastfeeding failure.

Functional and anatomical maturation of the gastrointestinal tract is strictly related to gestational age (GA); hence, premature infants are more prone to develop FD. Feeding intolerance (FI) is very common among preterm infants; clinical symptoms of FI (e.g. abdominal distension, vomiting, bilious gastric residuals, occult or gross bloody stools) are observed in nearly $29 \%$ of such neonates [1]. FI could represent an early sign of necrotizing enterocolitis (NEC), which is the most feared gastrointestinal complication of prematurity. Hence, FI often brings clinicians to withhold, decrease or discontinue enteral feeds, thus hampering the establishment of an adequate enteral nutrition and leading to a prolonged duration of both parenteral nutrition (PN) and central lines, with increased risks of such complications as liver cholestasis or sepsis[2].

The coordination between sucking, swallowing and breathing is usually achieved at 34-36 weeks GA; hence, preterm infants are usually fed via an intragastric tube, through intermittent boluses or continuously. Poor sucking and sucking-swallowing incoordination are the major causes of FD and breastfeeding failure among late preterm infants (GA 34-36 $6^{6 / 7}$ weeks), with an increased risk of hypoglycemia, excessive weight loss, hyperbilirubinemia, dehydration[3]. Due to FD, up to $27 \%$ of all late preterm infants need to be initially supplemented with intravenous fluids[4]; moreover, tube feeding is frequently required for feeding administration in the first days of life.

The abovementioned problems are infrequent in healthy term newborns. Term neonates developing FD

\footnotetext{
* Correspondence: silvia.martini4@gmail.com

Neonatology and Neonatal Intensive Care Unit, S.Orsola-Malpighi Hospital, Department of Medical and Surgical Sciences, University of Bologna, Bologna, Italy
}

such as poor sucking and/or vomiting need to be evaluated for pathological causes. Physical examination could aid to identify anatomical malformations possibly responsible for FD (e.g. cleft palate). FD and sleepiness can be due to hyperbilirubinemia, hypoglycemia or electisturbances[5], but could also subtend an erlying metabolic disease, such as hypothyroidism. lethargy and/or other clinical neurological signs (e.g. seizures, focal neurological signs, hypo- or hypertonia, fontanel, central apnoea) could address for central nervous system diseases (i.e. subarachnoid haemorFD and lischaemic stroke, metabolic encephalopathy etc.) invas lethargy could also represent a warning sign for tory distress, apnoea and bradycardia, temperature instability and increased capillary refill time[5]. Blood tests (including blood cells count, C-reactive protein, glucose, bilirubin, electrolytes, blood gas analysis) and cerebral ultrasound scan are useful tools to aid neonatologists in the differential diagnosis.

\section{Published: 30 September 2015}

\section{References}

1. Moore TA, Wilson ME, Schmid KK, Anderson-Berry A, French JA, Berger AM: Relations between feeding intolerance and stress biomarkers in preterm infants. J Pediatr Gastroenterol Nutr 2013, 57:356-62.

2. Embleton ND, Simmer K: Practice of parenteral nutrition in VLBW and ELBW infants. World Rev Nutr Diet 2014, 110:177-89.

3. Adamkin DH: Feeding Problems in the Late Preterm Infant. Clin Perinatol 2006, 33:831-7.

4. Wang ML, Dorer DJ, Fleming MP, Catlin EA: Clinical outcomes of nearterm infants. Pediatrics 2004, 114:372-6.

5. Webb T, Nugent M, Simpson P, Melzer-Lange M: Diagnostic findings in infants presenting to a pediatric emergency department for lethargy or feeding complaints. Pediatr Emerg Care 2014, 30:151-6.

doi:10.1186/1824-7288-41-S2-A21

Cite this article as: Corvaglia and Martini: Feeding difficulties during the neonatal period. Italian Journal of Pediatrics 2015 41(Suppl 2):A21. 